SLAC-PUB-14925

April 2012

\title{
Corrugated Pipe as a Beam Dechirper ${ }^{1}$
}

\author{
K.L.F. Bane and G. Stupakov, \\ SLAC National Accelerator Laboratory, Stanford, CA 94309, \\ USA
}

${ }^{1}$ Work supported by Department of Energy contract DE-AC02-76SF00515. 


\title{
Corrugated Pipe as a Beam Dechirper ${ }^{a}$
}

\author{
K.L.F. Bane and G. Stupakov \\ SLAC National Accelerator Laboratory, \\ Stanford University, Stanford, CA 94309
}

\begin{abstract}
We have studied the use of a metallic pipe with small corrugations for the purpose of passively dechirping, through its wakefield, a short, intense electron bunch. The corrugated pipe is attractive for this purpose because its wake: (i) has near maximal possible amplitude for a given aperture and (ii) has a relatively large oscillation wave length, even when the aperture is small. We showed how the corrugated structure can satisfy dechirping requirements encountered in the NGLS project at LBNL [1]. We found that a linear chirp of $-40 \mathrm{MeV} / \mathrm{mm}$ can be induced by an NGLS-like beam, by having it pass through a corrugated, metallic pipe of radius $3 \mathrm{~mm}$, length $8.2 \mathrm{~m}$, and corrugation parameters full depth $450 \mu \mathrm{m}$ and period $1000 \mu \mathrm{m}$. This structure is about 15 times as effective in the role of dechirper as an S-band accelerator structure used passively.
\end{abstract}

${ }^{\text {a }}$ Work supported by Department of Energy contract DE-AC02-76SF00515. 


\section{INTRODUCTION}

In a linac-based X-ray FEL, the beam, after leaving the gun, is accelerated in RF cavities and compressed in chicanes two or three times, and then accelerated once more, before finally entering the undulator for lasing. Typically the beam is not fully compressed in the final chicane, leaving it with an energy chirp that needs to be removed by the time it arrives at the undulator. In the Linac Coherent Light Source (LCLS) there are $550 \mathrm{~m}$ of S-band linac after the final chicane, and the wakefields of the cavities are used to passively cancel the chirp - to "dechirp" the beam. Some linac-based FEL's, however, use lower-frequency, superconducting L-band technology (e.g. TESLA XFEL in Hamburg [2] and the Next Generation Light Source (NGLS) in Berkeley [1]), where the wakefield is too weak to cancel the chirp. Others have soft X-ray options that need no acceleration beyond the final chicane (e.g. the PAL XFEL in Pohang [3]). In either case, the residual chirp can always be canceled actively by running the beam off-crest in a linac; however, this is an inefficient and costly option. There is interest, therefore, in developing an inexpensive, passive device that can be used as a dechirper.

A metallic beam pipe with small, periodic corrugations has been used as a model for roughness impedance and recently as a source of beam-driven terahertz radiation [4]. What makes it attractive as a dechirper (and what made it attractive as a $\mathrm{THz}$ radiator) is that one can choose parameters that yield a large wake amplitude - by taking a small pipe aperture - and at the same time have a wake that oscillates with a relatively long wavelength. Note that an object that behaves similarly - a beam pipe with a thin dielectric layer — was proposed in 1990 by Rosing and Simpson to be used as a "wakefield silencer" (a dechirper) [5].

In this report we begin by reviewing basic analytic properties of a metallic 
pipe with small corrugations. We then apply the results to designing an example device to satisfy dechirper requirements for the NGLS beam. We also include a numerical simulation, where we use I. Zagorodnov's time-domain, finite difference wakefield solver, ECHO [6]. We compare analytical and numerical results. We provide a discussion section, and finally a conclusion.

\section{THEORY}

Consider a short, ultra-relativistic bunch of electrons passing on-axis through a periodic (cylindrically-symmetric) metallic structure with small corrugations. Let the pipe radius be $a$ and the corrugation properties be: depth $\delta$, period $p$, and gap $g$, with $\delta, p \ll a$ (see Fig. 1). We further require $\delta \gtrsim p$, in which case we designate the structure a "steeply corrugated" structure. Then the beam excites one strong fundamental mode with a frequency that is far above the beam pipe cut-off. For this mode the wave number, $k$, is well approximated by $[7,8]$

$$
k=\sqrt{\frac{2 p}{a \delta g}}
$$

and the mode loss factor by

$$
\varkappa=\frac{Z_{0} c}{2 \pi a^{2}},
$$

where $Z_{0}=377 \Omega$ and $c$ is the speed of light. The point charge wake is simply given by

$$
W(s)=2 \varkappa H(s) \cos k s,
$$

with the unit step function $H(s)=1(0)$ for $s>(<) 0$, and the distance between driving and test particles given by $s$ (with $s>0$ indicating a point behind the driving particle).

The wake at the origin for any round, periodic structure of minimum aper- 


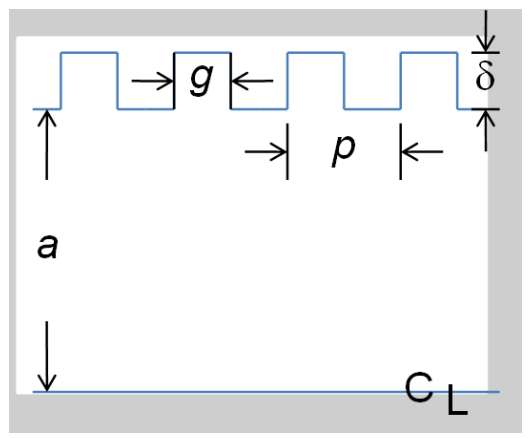

FIG. 1. A sketch of the corrugated structure.

ture $a$ is

$$
W\left(0^{+}\right)=2 \sum_{n} \varkappa_{n}=\frac{Z_{0} c}{\pi a^{2}},
$$

where $\varkappa_{n}$ is the loss factor of mode $n$. This is true for a smooth resistive pipe [9], for a metallic pipe with a thin dielectric layer [10], for a disk-loaded accelerator structure [11], and for the corrugated pipe. It is believed to be a general property of wakefields in structures with cylindrical symmetry. What makes the corrugated pipe unique and attractive as a dechirper is: (i) it has only one significant mode, with the near maximal $\varkappa$ for a given aperture (see Eq. 2), and (ii) one can have $a$ small, for a strong interaction, and still choose corrugation parameters to give a relatively low $k$.

For a bunch with longitudinal distribution $\lambda(s)$, the bunch wake is given by the convolution

$$
W_{\lambda}(s)=-\int_{0}^{\infty} W\left(s^{\prime}\right) \lambda\left(s-s^{\prime}\right) d s^{\prime}
$$

here a negative value of $s$ is at the front of the bunch, and a negative value of $W_{\lambda}(s)$ means energy loss. The average of the bunch wake is the bunch loss factor

$$
\varkappa_{\lambda}=-\left\langle W_{\lambda}\right\rangle \equiv-\int_{-\infty}^{\infty} W_{\lambda}(s) \lambda(s) d s
$$


In the case of a Gaussian bunch of length $\sigma_{z}$

$$
\varkappa_{\lambda}=\frac{Z_{0} c}{2 \pi a^{2}} e^{-k^{2} \sigma_{z}^{2}}
$$

for a very short bunch $\left(k \sigma_{z} \ll 1\right), \varkappa_{\lambda} \approx Z_{0} c / 2 \pi a^{2}$. For a pipe of length $L$, the wake induced energy change is given by $U_{\lambda}(s)=e^{2} N W_{\lambda}(s) L$, with $e N$ the bunch charge, and the average energy loss in the bunch is $U_{\text {loss }}=e^{2} N \varkappa_{\lambda} L$.

Note that our analytical results are valid under the conditions: $\delta / a, p / a \ll 1$ and $\delta \gtrsim p$ (a steeply corrugated structure). If we e.g. somewhat violate the last condition, the mode frequency will be higher and the excitation (mode loss factor) lower than the analytical values [7]. In the regime of a "shallowly corrugated" structure $(\delta \ll p)$ the dominant mode is gone, and the structure wake is completely different [12].

Note also that the results given here are steady-state results, assuming the beam travels through a truly periodic structure. With a finite-length pipe there is also a transient response, induced when the beam enters the pipe. After the beam has traveled a distance on the order of the catch-up distance,

$$
z_{c u}=\frac{a^{2}}{2 \sigma_{z}}
$$

with $\sigma_{z}$ the rms bunch length, the steady-state behavior dominates and this component can be ignored. We will find that for reasonable parameters for a dechirper this will indeed be the case. The second effect of a finite-length pipe - that due to the high group velocity in the radiation pulse - is discussed in the following section, and will also be shown to be unimportant for the dechirper.

Finally, note that if the beam traverses the corrugated pipe off-axis, a strong dipole mode of almost the same frequency as the fundamental mode will be excited [8], which can generate an unwanted transverse kick to the beam and lead to instability. We can estimate the strength of the effect. Assuming a 
uniform bunch distribution of length $\ell$, the instability strength at the tail of the bunch can be characterized by the dimensionless parameter [13]

$$
\Upsilon=\frac{e^{2} N W_{x}^{\prime}(0) \ell \beta_{x} L}{2 \mathcal{E}}
$$

with $W_{x}^{\prime}$ the slope of the dipole wakefield, $\beta_{x}$ the average of the optical beta function, and $\mathcal{E}$ the beam energy. For the corrugated pipe $W_{x}^{\prime}(0)=$ $2 Z_{0} c /\left(\pi a^{4}\right)[8]$. For stability $\Upsilon$ should be small compared to 1.

\section{Radiation Pulse}

The beam, in its passage through the corrugated pipe, generates a radiation pulse [4]. When the bunch leaves the structure, it will directly be followed by a relatively long, multi-cycle pulse. The radiation pulse properties, though maybe having no direct bearing on the dechirping, may nevertheless be used as a diagnostic for such a device in a real machine, and may affect its implementation. For example, in the NGLS the pulse repetition rate is very high and one needs to make sure that resistive wall heating, caused by the pulse, does not become a problem. We therefore present here a few of the pulse properties. For more details, the reader is referred to Ref. [4].

As it traverses the corrugated pipe the beam generates a radiation pulse with group velocity $v_{g}$ given by

$$
\left(1-\frac{v_{g}}{c}\right)=\frac{4 \delta g}{a p}
$$

at the end of a structure of length $L$ the pulse will have length

$$
l_{p}=\frac{4 \delta g L}{a p} .
$$

Note that Eq. 11 implies that the simple equation for the wake, Eq. 3, cannot

be correct for a finite-length structure; a test particle at $s / c>l_{p} / v_{g}$ does not 
encounter the pulse before it has left the structure, and thus sees no wake. However, for dechirper-type of parameters, we shall see that $s$ of interest is small compared to $l_{p}$, and this effect can be ignored.

The pulse will be hollow, with the fields varying linearly with $r$ : they can be written as, $E_{r}(r)=H_{\phi}(r)=H_{0} r / a$, with $E_{r}$ the radial electric field, $H_{\phi}$ the azimuthal magnetic field, and $H_{0}$ a constant. Taking the pulse energy equal to the wake energy, we can estimate the maximum $E_{r}(a)$ for the pulse:

$$
\hat{E}_{r}(a)=\frac{2 e N}{a} \sqrt{\frac{Z_{0} c \varkappa L}{\pi l_{p}}} .
$$

With resistive walls, the wake energy lost by the bunch equals the energy in

the pulse plus the energy deposited in the walls. The dissipation length-the distance along the pipe over which the fields drop by $1 / e$-is

$$
L_{D}=\frac{a}{4 \zeta^{\prime}(1+2 \delta / p)}
$$

with $\zeta^{\prime}=(k c / 8 \pi \sigma)^{1 / 2}$, and $\sigma$ the conductivity of the metal walls. The fraction of wake energy that is deposited in the walls is $(1-\eta)$, with

$$
\eta=\frac{L_{D}}{L}\left(1-e^{-L / L_{D}}\right)
$$

the power lost in the walls, $\mathcal{P}_{r w}=f_{\text {rep }}(1-\eta) N U_{\text {loss }}$, with $f_{\text {rep }}$ the beam repetition rate.

\section{DECHIRPER}

After the final bunch compression in a linac-based FEL the tail of the beam is typically at higher energy than the head. In this case we want a dechirper that is capacitive-i.e. one in which the bunch tail loses more energy than the head - which implies we need a bunch that is short compared to the wavelength 
of the mode of the structure, i.e. we need $k \sigma_{z} \ll 1$. If this condition is satisfied, then Eq. 5 can be approximated

$$
W_{\lambda}(s) \approx-2 \varkappa \int_{-\infty}^{s} \lambda(x) d x
$$

For a Gaussian bunch with $k \sigma_{z} \ll 1, W_{\lambda}(s)=-\varkappa\left[1+\operatorname{erf}\left(\mathrm{s} / \sqrt{2} \sigma_{\mathrm{z}}\right)\right]$, and the chirp introduced by the wake is monotonic but not linear. In the case of a uniform distribution of length $l$ (with $k l / 3.5 \sim k \sigma_{z} \ll 1$ ), Eq. 15 becomes $W_{\lambda}(s) \approx-2 \varkappa s / l$, and the chirp is linear. For a uniform bunch distribution the chirp at end of a pipe of length $L$ is given by

$$
h=-\frac{2 \varkappa e N L}{l} \text {. }
$$

For linear dechirping we need a uniform bunch distribution. In real situations, even if the bunch is uniform over the core of the distribution, it will have horns or smooth transitions to zero at the head and tail. In such a case the dechirping will still be linear over the uniform core; however, only the charge within the uniform region will contribute to the linear chirp. In this case Eq. 16 should be replaced by

$$
h=-2 \frac{\varkappa \alpha e N L}{l},
$$

with $\alpha$ the fraction of charge in the uniform part of the bunch distribution.

Finally, note that if we needed to induce a chirp of the opposite sign (if e.g. we changed from under- to over-compression in the final chicane of the LCLS), our structure would be of questionable utility. In this case we would need to choose pipe parameters giving $k \sigma_{z} \gg 1$, resulting in an inductive wake. Then, in the case of a Gaussian bunch distribution, $W_{\lambda}(s) \sim s e^{-s^{2} / 2 \sigma_{z}^{2}}$, which is linear over the core of the bunch, but non-linear with slopes of the wrong sign at bunch head and tail. In the case of a uniform bunch distribution, the wake would be $W_{\lambda}(s) \sim[\delta(s-\ell / 2)-\delta(s+\ell / 2)]$ (assuming the head and tail are at $\mp \ell / 2$ ), and the wake is zero over the bunch core. 


\section{EXAMPLE CALCULATION}

For an example calculation we consider dechirping requirements for the NGLS project at LBNL. In a typical mode of operation the beam is operated at currents of 500-600 A, where the bunch charge is $e N=300 \mathrm{pC}$ [14]. Just past the second bunch compressor the pulse shape is nearly uniform $(\sim 75 \%$ of the beam is within the uniform core), with length $\ell=150 \mu \mathrm{m}$; the energy $\mathcal{E}=350 \mathrm{MeV}$, and there is a rather linear chirp (over the beam core) of $+40 \mathrm{MeV} / \mathrm{mm}$ (the beam tail is at higher energy than the head). After the compressor the beam is accelerated to $1.8 \mathrm{GeV}$ in a superconducting linac. To cancel the chirp with RF alone, the beam would need to run at $25^{\circ}$ behind crest, resulting in $10 \%$ loss in efficiency of acceleration.

The dechirping can be done passively in a corrugated pipe. The required chirp is $h=-40 \mathrm{MeV} / \mathrm{mm}$. We assume a uniform charge distribution (with fraction of charge $\alpha=0.75$ in the uniform core), with bunch length and charge given above. How do we choose the structure parameters? Let us say we want to limit the relative size of the 3rd order term in the chirp at the end of the uniform bunch of length $\ell$ to the value $\epsilon$; i.e. we want $(k \ell)^{2} / 2=\epsilon$. Using Eq. 1, this implies that we choose $a$ to be

$$
a=\frac{\ell}{\sqrt{(g / p)(\delta / a) \epsilon}} .
$$

The ratio $(\delta / a)$ needs to be small, for the analytical formulas to be valid, but not too small, in order to keep $k$ relatively small; we take $(\delta / a)=0.15$. For low $k$, we would also want $(g / p) \approx 1$ (see Eq. 1). However, this would result in very thin irises, which is not desirable. So we choose $(g / p)=0.75$. We want the 3rd order term in the chirp to be small and take $\epsilon=0.02$. Then the proper choice of $a=3 \mathrm{~mm}$ and $\delta=450 \mu \mathrm{m}$. The period $p$ should not be large compared to $\delta$ and should be small compared to $a$; we take $p=1000 \mu \mathrm{m}$; then 
$g=750 \mu \mathrm{m}$. With these parameters the chirp can be achieved (see Eq. 17) with a pipe of length $L=6.65 \mathrm{~m}$. These parameters yield (Eq. 1) $k=1.4 \mathrm{~mm}^{-1}$; $k \ell=0.21$, and average energy loss in the bunch $U_{\text {loss }} \approx e N \varkappa L=4.0 \mathrm{MeV}$ or, equivalently, the total energy lost is $1.2 \mathrm{~mJ}$.

The catch-up distance $z_{c u}=10 \mathrm{~cm}$ (taking $\sigma_{z}=150 \mu \mathrm{m} / 3.5$ ), which is small compared to the structure length; thus one can ignore the transient response of the structure. For the radiation pulse, $\left(1-v_{g} / c\right)=0.45$, and the pulse length $l_{p}=3.0 \mathrm{~m}$. Since $s / l_{p}$ for $s$ of interest is very small, the modification of the wake due to the finite pulse length, discussed earlier, is small and can also be ignored. The maximum of $E_{r}$ near the boundary, $\hat{E}_{r}(a)=2.5 \mathrm{MV} / \mathrm{m}$.

For the radiation pulse [assuming $\sigma=5.4 \times 10^{17} / \mathrm{s}(\mathrm{Cu})$ ], the dissipation length for wall losses $L_{D}=2.2 \mathrm{~m}$, and the fraction of energy deposited in the walls $(1-\eta)=0.68$. The power dissipated in the walls, assuming a repetition rate $f_{\text {rep }}=1 \mathrm{MHz}$ is $122 \mathrm{~W} / \mathrm{m}$, which should not be difficult to manage.

To estimate the strength of the dipole mode we use Eq. 9, taking $\beta_{x}=10 \mathrm{~m}$. We obtain as strength parameter at the tail of the bunch, $\Upsilon=3.8$. For stability, this parameter should be small compared to 1 . The fact that it is not small and also not large implies that the transverse instability will be an important issue for such a dechirper if used in the NGLS, but one that is manageable. The parameters and selected properties of the model dechirper, meant for the NGLS example problem, is given in Table I.

\section{NUMERICAL SIMULATION}

So far all the results have been analytical, using formulas meant to be valid for small parameters. To obtain accurate results for our specific structure, we have also performed numerical simulations using I. Zagorodnov's time domain, finite difference computer program ECHO, a code for finding wakefields in 2D 
TABLE I. Example NGLS dechirper parameters. Also given are the analytical values of wavenumber $k$, mode loss factor $\varkappa$, fraction of wake energy that ends up in the walls, $(1-\eta)$, and resistive wall heating per unit length, $\mathcal{P}_{r w} / L$. We have assumed the pipe is $\mathrm{Cu}$; that $e N=300 \mathrm{pC}$ and $f_{r e p}=1 \mathrm{MHz}$.

\begin{tabular}{||c|c|c|c|c||c|c|c|c||}
\hline \hline$a[\mu \mathrm{m}]$ & $\delta[\mu \mathrm{m}]$ & $p[\mu \mathrm{m}]$ & $g[\mu \mathrm{m}]$ & $L[\mathrm{~m}]$ & $k\left[\mathrm{~mm}^{-1}\right]$ & $\varkappa[\mathrm{MV} / \mathrm{nC} \cdot \mathrm{m}]$ & $(1-\eta)$ & $\mathcal{P}_{r w} / L[\mathrm{~W} / \mathrm{m}]$ \\
\hline \hline 3000 & 450 & 1000 & 750 & 6.65 & 1.4 & 2.0 & 0.68 & 122. \\
\hline \hline
\end{tabular}

(cylindrically symmetric) structures [6]. Specifically, our goal here is to verify that - for an NGLS-type bunch - a linear energy chirp is induced, and to find the length of pipe needed to obtain the correct chirp amplitude.

The program ECHO is meant to be run using Gaussian driving bunches. Thus the wake for the flat-top bunch needs to be calculated in two steps. In the first step, ECHO is run for the structure of Table I using a short Gaussian driving bunch $\left(\sigma_{z 0}=10 \mu \mathrm{m}\right)$, calculated out to $s=250 \mu \mathrm{m}$ behind bunch center, in order to generate a "Green function" wake. In the second step the Green function is convolved with the desired flat-top bunch distribution.

The mesh size for the ECHO run was $2 \mu \mathrm{m}$ in both radial and longitudinal directions. The run took several hours on a Windows desktop computer. The results are shown in Fig. 2 (the blue curve). The red dashes give the analytical approximation, $W_{\lambda 0} L=-\varkappa L\left(1+\operatorname{erf}\left(\mathrm{s} / \sqrt{2} \sigma_{\mathrm{z} 0}\right)\right)$, with $\varkappa, L$, given in Table I. The agreement between the two results is very good until $\sim 10 \mu \mathrm{m}$ behind bunch center. Note also that the numerically obtained wake loss of the bunch agrees with the expected result, $U_{\text {loss }}=W\left(0^{+}\right) / 2=Z_{0} c /\left(2 \pi a^{2}\right)$ (Eq. 4$)$, to $4 \%$; this is an accuracy check on the numerical results.

Behind $s=10 \mu \mathrm{m}$, however, the numerical result begins to deviate and become less (in amplitude) than the analytical one. This deviation is not due to the curvature in the wake, which we ignored in the analytical calculation, 


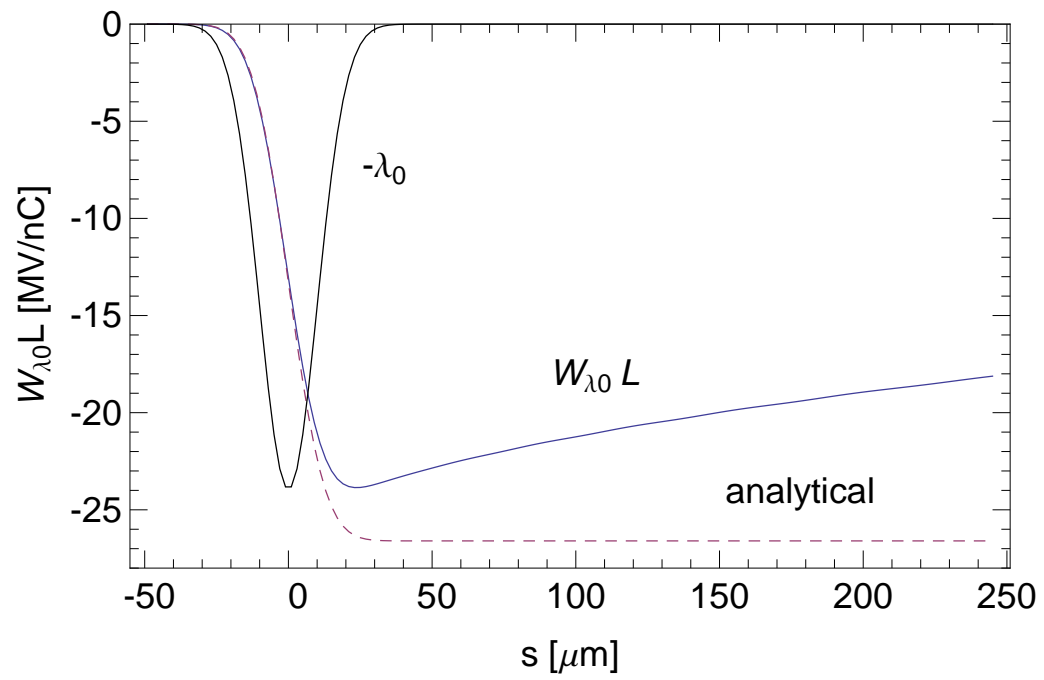

FIG. 2. Dechirper for NGLS, Green function: wake of $\sigma_{z 0}=10 \mu \mathrm{m}$ bunch (blue). The dashed, red curve gives the analytical approximation $W_{\lambda 0} L=$ $-\varkappa L\left[1+\operatorname{erf}\left(\mathrm{s} / \sqrt{2} \sigma_{\mathrm{z} 0}\right)\right]$, with $L, \varkappa$, given in Table I. The driving bunch shape $\lambda_{0}$, with the head to the left, is given in black.

since this component contributes only a few percent at $s \sim 100 \mu \mathrm{m}$. We believe this discrepancy is due to the effect of higher order modes in the structure. Repeating the ECHO run, but this time finding the wake to $6 \mathrm{~mm}$ behind the bunch, we obtained a wake with frequency and amplitude respectively $8 \%$ higher and $27 \%$ lower than our analytical expectations. To verify these results we also ran KN7C, a program that uses field matching to find the longitudinal modes in a periodic, disk-loaded structure [15]. For KN7C the fundamental mode frequency and loss factor are respectively $9 \%$ higher and $32 \%$ lower than the analytical expectations, in good agreement with the time-domain, ECHO results. These result is not surprising: the analytical formulas were meant for small corrugations, and our corrugations are apparently not sufficiently small. 
For the second step of obtaining the wake for the NGLS bunch, the Green function wake was convolved with a model of the NGLS bunch shape. The actual NGLS bunch shape consists of $100 \mu \mathrm{m}$ of a (relatively) flat-top core, a horn in front, and a Gaussian decay (with $\sigma \sim 20 \mu \mathrm{m}$ ) in back; about $75 \%$ of the beam charge is in the core. We take as model of the NGLS bunch shape the following:

$$
\lambda(s)=\frac{1}{\ell+\sqrt{2 \pi} \sigma_{b}}\left\{\begin{array}{rll}
1 & : & |s|<\ell / 2 \\
e^{-s^{2} / 2 \sigma_{b}^{2}} & : & |s|>\ell / 2
\end{array}\right.
$$

where $\ell=150 \mu \mathrm{m}$ and $\sigma_{b}=20 \mu \mathrm{m}$. For convolution we perform the integral

$$
W_{\lambda}(s)=-\int_{-\infty}^{\infty} W_{\lambda 0}\left(s^{\prime}\right) \lambda\left(s-s^{\prime}\right) d s^{\prime} .
$$

The resulting bunch wake is shown in Fig. 3 (the blue curve). We see a nearly perfectly linear chirp over the uniform bunch core of the distribution. The chirp, however, is $h=-32.5 \mathrm{MeV} / \mathrm{mm}, 19 \%$ below our requirement, due to the deviation between numerical and analytical results in the Green function noted above. Thus, to obtain a chirp of $h=-40 \mathrm{MeV} / \mathrm{mm}$ with this structure, the dechirper needs to be lengthened to $L=8.2 \mathrm{~m}$.

\section{DISCUSSION}

A smooth, resistive pipe can also serve as a dechirper. To compare its effectiveness with that of the corrugated pipe note that, in both cases, the point charge wake at the origin $W\left(0^{+}\right)=Z_{0} c /\left(\pi a^{2}\right)$, where $a$ is the radius of the pipe. The first zero-crossing of the wake for the corrugated pipe is at $s=\pi /(2 k)=900 \mu \mathrm{m}$. However, for the resistive pipe the first zero-crossing occurs at $s \sim s_{0}=\left(c a^{2} / 2 \pi \sigma\right)^{1 / 3}$, with $\sigma$ the resistivity of the metal [9]. For $a=3 \mathrm{~mm}$ (the same aperture as the corrugated pipe), $s_{0}=9 \mu \mathrm{m}(\mathrm{Cu})$, 


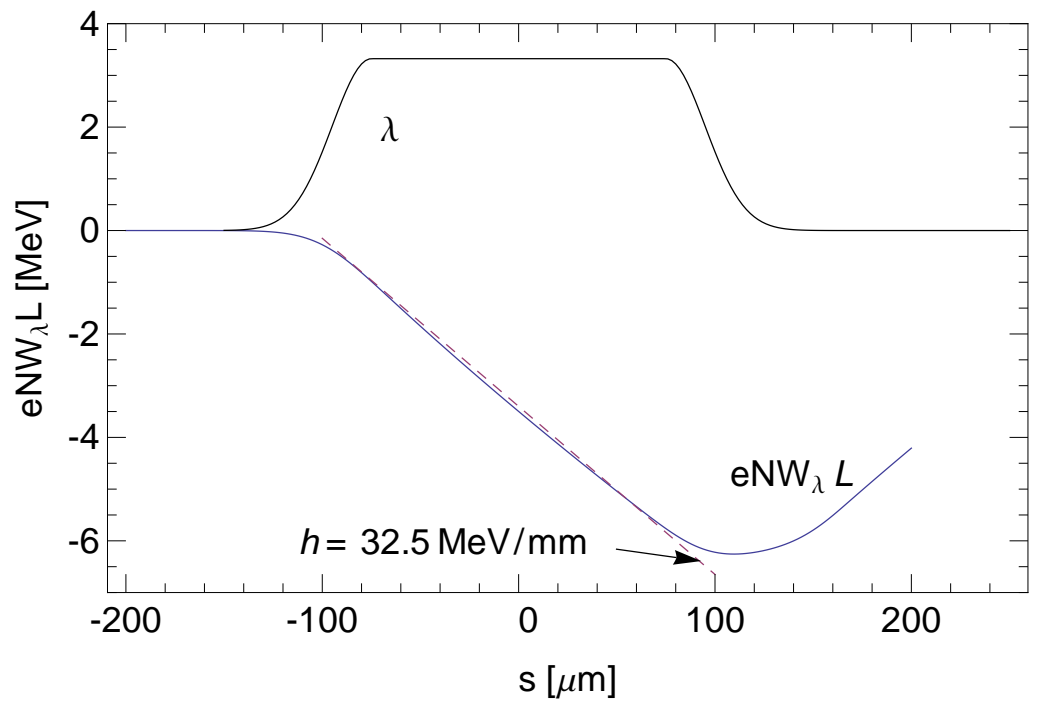

FIG. 3. Dechirper for NGLS: wake of model of NGLS bunch distribution (blue). The dashed, red curve represents a chirp $h=-32.5 \mathrm{MeV} / \mathrm{mm}$. The bunch shape, with the head to the left, is given in black.

$32 \mu \mathrm{m}$ (stainless steel). Thus, because of the very short distance to the first zero-crossing in the wake, the smooth resistive pipe is much inferior to the corrugated pipe in the role of a dechirper.

For an S-band accelerator structure, like that of the SLAC linac, the shortrange wake can be approximated by [11]

$$
W(s)=\frac{Z_{0} c}{\pi a^{2}} H(s) e^{-\sqrt{s / s_{1}}},
$$

with $s_{1}=0.41 a^{1.8} g^{1.6} / L^{2.4}$. To first order, the relative dechirping effectiveness (dechirping per unit length) compared to the corrugated pipe is given by the ratio of $a^{-2}$ for the two structures. For the SLAC structure $a \sim 1.2 \mathrm{~cm}$, which implies it is about a factor 15 less effective than the corrugated dechirper.

But it is not just the aperture; the shape of the S-band structure is also not optimal for the role of dechirper. If in a Gedanken-experiment the S- 
band structure were scaled in dimensions by a factor 0.26 to give the same aperture [and same $W\left(0^{+}\right)$], the resulting $W(s)$ would have a relatively rapid droop, by $55 \%$ at $s=150 \mu \mathrm{m}$. When convolved with the model NGLS charge distribution, the result is a linear energy chirp only $2 / 3$ that of the corrugated pipe (per unit length of structure), and includes also a significant non-linear chirp component. The corrugated pipe clearly makes a better dechirper.

For an adjustable dechirper one can consider using parallel metallic plates with corrugations, and have the distance between the plates adjustable, where the strength of interaction changes as $a^{-2}$, with $a$ the the half-distance between the two plates. Earlier studies have found that a mode similar to that in the round pipe is generated by this configuration, though for a given aperture the excitation is a factor $\pi^{2} / 16$ weaker and the mode wave number a factor $1 / \sqrt{2}$ lower (see e.g. [16]). Because of the added flexibility in chirp control, the flat geometry may be a better option than the round geometry considered in this report.

For this report we have used corrugations with sharp edges, mainly for illustrative purposes. To avoid high electric fields on the surface it is normally desirable to have smoothed corners instead. With the right choice of smooth contour the dechirping properties of the pipe will not change (we've done tests with ECHO). Specifically, if we consider the original, rectangular corrugation to vary between $y= \pm \delta / 2$, then the smoothed version needs to keep the oscillation period and the area under the positive and negative lobes of the oscillation unchanged.

Finally, it should be noted that one can dechirp as effectively by using a metallic pipe with a thin dielectric liner (with no corrugations) instead of the corrugated metallic pipe described here - the wake effect is equivalent [10]. With $\delta$ the thickness of dielectric layer, the same formula holds for the loss factor $\varkappa$, and the wave number $k=\sqrt{2 \epsilon /(\epsilon-1) a \delta}$, with $\epsilon$ the dielectric con- 
stant. There may be advantages in using a dielectric, such as a dielectric may be more robust to damage by surface electric fields. It will be such practical considerations that will decide which of these two options is better.

\section{CONCLUSIONS}

We have studied the use of a metallic pipe with small corrugations for the purpose of passively dechirping, through its wakefield, a short, intense electron bunch. For linear dechirping the bunch needs to have a uniform charge distribution, or at least a uniform core. What makes the corrugated pipe unique and attractive as a dechirper is that it has only one significant mode, with near maximal possible interaction strength (mode loss factor $\varkappa$ ) for given aperture $a$; and that one can have both large $\varkappa$, by choosing small $a$, and a relatively low mode wave number $k$, by adjusting the corrugation parameters.

Another attractive feature of the corrugated pipe is that its wake properties can be well approximated by simple analytical formulas. We presented formulas for designing a dechirper using the corrugated pipe and, as practical example, applied them to parameters for the NGLS project. We then performed numerical simulations, using the computer program ECHO. We found that, indeed, a linear chirp was induced by a uniform bunch distribution; the size of the numerical chirp, however, was $20 \%$ lower than the analytical approximations.

A specific result of this report is that a linear chirp of $-40 \mathrm{MeV} / \mathrm{mm}$ can be induced by an NGLS-like beam, by having it pass through a corrugated, metallic pipe of radius $3 \mathrm{~mm}$, length $8.2 \mathrm{~m}$, and corrugation parameters full depth $450 \mu \mathrm{m}$ and period $1000 \mu \mathrm{m}$. This structure is about 15 times as effective in the role of dechirper as an S-band accelerator structure used passively. 


\section{ACKNOWLEDGEMENTS}

The authors thank P. Emma and K.-J. Kim for encouraging us to do this work, and M. Venturini for supplying us with parameters and other information on the NGLS project.

[1] J. Corlett et al, in Proc. of IPAC'10, Kyoto, Japan, 2010, pp. 2639-41.

[2] H. Weise, in Proc. of EPAC'04, Lucerne, Switzerland, 2004, pp. 11-16.

[3] M. Yoon et al, in Proc. of FEL08, Gyeongju, Korean, 2008, pp. 218-21.

[4] K. Bane and G. Stupakov, Nucl. Inst. Meth. A 677 (2012) 67-73.

[5] M. Rosing and J. Simpson, "Passive momentum spread reduction, the wakefield silencer," ANL Report WF-144, April 1990.

[6] I. Zagorodnov and T. Weiland, Phys. Rev. ST Accel. Beams 8 (2005) 042001.

[7] K. Bane and A. Novokhatski, "The resonator impedance model of surface roughness applied to the LCLS parameters," LCLS-TN-99-1, SLAC, 1999.

[8] K. Bane and G. Stupakov, in Proc. of LINAC00, Monterey, CA, 2000, pp. 92-94.

[9] K. Bane and M. Sands, Proc. of Micro Bunches Workshop, AIP Conf. Proc. 367, Upton, NY, 1995, pp. 131-149.

[10] A. Mosnier and A. Novokhatski, in Proc. of PAC97, Vancouver, Canada, 1997, pp. $1661-63$.

[11] K. Bane et al, in Proc. of ICAP'98, Monterey, CA, 1998, pp. 137-39.

[12] G. Stupakov, Proc. of Physic and Science of the X-Ray Free -Electron Laser, Arcidosso, Italy, 2000, p. 141.

[13] A. Chao et al, Nucl. Inst. Meth. 178 (1980) 1-8.

[14] M. Venturini, private communication. 
[15] E. Keil, Nucl. Inst. Meth. 100 (1972) 419-427.

[16] K. Bane and G. Stupakov, Phys. Rev. ST Accel. Beams 6 (2003) 024401. 